\title{
LICENCIAS POR MATERNIDAD Y PATERNIDAD PARA ESTUDIANTES DE UNIVERSIDADES ARGENTINAS DESDE UNA PERSPECTIVA DE GÉNERO
}

Rocío Riesco

Facultad de Derecho, Universidad de Buenos Aires

http://dx.doi.org/10.5209/NOMA.56610

Resumen: en el sistema universitario argentino, la licencia por maternidad y paternidad para estudiantes no es una problemática frecuente en las investigaciones académicas. Sin embargo, desde el año 2011 el tema ha empezado a adquirir un rol más visible a partir de la reciente creación de un marco normativo que implementa un sistema de licencias dirigido al sector estudiantil con el objetivo de promover cierta equidad en el derecho al cuidado. El presente trabajo se propone, entonces, ahondar en los avances en materia de género que conlleva la incorporación de estos beneficios para quienes estudian en algunas universidades de la Argentina.

Palaras clave: derecho al cuidado - licencias parentales- universidades - género

Abstract: in the Argentine university system, student maternity and paternity leave is not a frequent subject of academic research. However, recently the topic has become more visible since a regulatory framework was introduced in 2011 implementing a system of maternity/paternity for the student body with the aim of promoting certain equity in the right to care. The present article attempts therefore to examine the progress that the incorporation of these benefits for university alumni represents in terms of gender equality in Argentina.

Keywords: right to care - parental leave - universities - gender

\section{Introducción}

Las licencias parentales destinadas a estudiantes de las universidades argentinas son una problemática reciente. En cambio, existen avances sustantivos en otros aspectos del problema: las licencias y el sistema de prestaciones que cuentan las trabajadoras formales asalariadas y su vínculo con el mercado laboral, la relación entre las licencias y los derechos de los niños/as, el derecho laboral de las madres trabajadoras, así como el avance en materia legislativa y los proyectos de ley con estado parlamentario.

Asimismo, si bien no se trata de un tema frecuente en la investigación académica, ha empezado a adquirir un rol más visible en la última década a partir de la reciente creación de un marco normativo que implementa un sistema de licencias para los y las estudiantes universitarios/as. Como parte de esta tendencia, dediqué los últimos años al proyecto "Una aproximación socio jurídica a las respuestas de 
las universidades nacionales a las necesidades de cuidado en la primera infancia. Un estudio de caso centrado en el sistema de licencias y de servicios de cuidado para los/as estudiantes universitarios", dirigido por Carla Zibecchi y financiado por una Beca Estímulo UBACyT 2015$2016^{1}$.

Este artículo pretende difundir algunos avances de ese proyecto y, en especial, plantear con mayor profundidad las implicancias de género que conlleva la implementación de sistemas de licencias maternales y parentales para los y las estudiantes universitarios. A tal efecto, se focaliza en la normativa establecida por las entidades públicas nacionales desde el año 2011 hasta el presente, ya que se formulan en ese período un conjunto de reglamentaciones orientadas a la atención de esta problemática. Hasta entonces, las situaciones de embarazo estudiantil y la consecuente necesidad de cuidado de los niños, las niñas y los núcleos familiares, estaban exentos de protección reglamentaria y eran asumidos, en gran medida, por las mujeres. Así, las posibilidades de desarrollo de las mujeres en etapa universitaria quedaban restringidas $e$, incluso, anuladas por la necesidad de cuidado del otro/a. Los cambios reglamentarios producidos en algunas universidades argentinas que habilitan licencias maternales y parentales parecen subsanar, en parte, estas restricciones y abren un espacio de expectativa en pos de la igualdad social y, en especial, de género.

En concordancia con estos planteos, se aborda, primer lugar, el cuidado y la maternidad desde una visión socio jurídica, mostrando cómo las licencias por maternidad y paternidad conforman una manera de regular el cuidado que viene a resguardar y a proteger la maternidad.

En segundo lugar, se analizan los sistemas de licencias existentes para estudiantes universitarios, a partir de la creación de un reciente marco normativo por parte de algunas universidades nacionales (Tucumán 2007; Córdoba 2011; La Pampa 2011; Salta 2012; Cuyo 2013; La Rioja 2015 y Entre Ríos 2015) y se abordan los cambios que se introdujeron en cada caso, con su respectivo fundamento jurídico-normativo.

Por último, se describe el régimen implementado por la Universidad Nacional de La Pampa (UNLPam), ya que es uno de los primeros y más completos sistemas de los que se registran en la Argentina. Se discuten los beneficios que conlleva, como así también sus implicancias prácticas para el desempeño académico del estudiantado. La recopilación de los datos se realizó mediante la consulta y el análisis de

\footnotetext{
1 Este trabajo es un avance parcial de ese proyecto que integra, además, el proyecto UBACYT Interdisciplinario en Formación: "Familias y necesidades de cuidado en contextos de marginalidad social. Una mirada sociojurídica en torno a las respuestas de las políticas sociales y las sentencias judiciales", radicado en el Instituto Ambrosio L. Gioja de la Facultad de Derecho de la Universidad de Buenos Aires, desde 2014 hasta el 2017.
} 
los documentos disponibles en forma remota en cada universidad y, en el caso específico de la UNLPam, se complementó con entrevistas personales al miembro de la agrupación estudiantil que presentó el proyecto a consideración del Consejo Superior de la universidad y, también, a los responsables de su aplicación.

\section{Maternidad y derecho al cuidado: una aproximación socio-jurídica}

El concepto de cuidados encierra variadas dimensiones y es atravesado por distintos conflictos ideológicos que dificultan el consenso en cuanto a su definición como objeto de estudio. A partir de la década del ochenta del siglo pasado, surge como categoría analítica, adquiere cada vez mayor importancia en el pensamiento feminista e influye los estudios en la esfera de la academia, donde la abundancia de escritos existentes a la fecha demuestra de la centralidad de la temática (Borderías, Carrasco y Torns, 2011).

Con independencia de las diferencias disciplinares que existen en relación al tema, resulta importante destacar, según señala Esquivel (2015), que las contribuciones académicas han permitido desnaturalizar el cuidado como lo propio de las mujeres y desplazarlo del ámbito privado para hacerlo público y politizable. Se trata de un concepto que entrelaza lo económico -la forma en que las economías se benefician del trabajo de cuidados que no es ni reconocido ni remunerado-, lo social -las relaciones de clase y género- y lo político -los diferentes actores que demandan, sostienen o implementan políticas públicas que, ya sea directa $o$ indirectamente, moldean la prestación y recepción de cuidados.

Actualmente, no existe un reconocimiento amplio de la importancia del cuidado para la vida. Pautassi (2007), en ese contexto, propone reconocer al cuidado como un derecho humano universal. Según la autora, para la consagración del derecho al cuidado como derecho universal resulta necesario no sólo la promoción de una oferta de cuidado, sino también la universalización de la responsabilidad, la tarea y la asignación de los recursos materiales para realizarlo (Pautassi, 20072013), de modo tal de asegurar su reconocimiento como un derecho universal e inalienable a cuidar, ser cuidado y a cuidarse. Así se daría un primer paso para lograr distribuir las responsabilidades de cuidado entre todos los miembros de la sociedad y no depositarlas, como ocurre en general, sólo en las mujeres (Gherardi y Zibecchi, 2011).

Ahora bien, a pesar de que en los estudios de género y la literatura feminista se hacen numerosas referencias y explicitaciones conceptuales en torno al derecho al cuidado (de niños, niñas, adolescentes, enfermos, adultos mayores) y a cuidar (se), éste no ha sido aún incluido en los Tratados y Pactos internacionales de Derechos Humanos. No obstante, suele afirmarse que este derecho está 
incorporado en función de lo normado en cada uno de los derechos sociales incluidos, que van desde el derecho a una alimentación de calidad y en cantidad suficiente hasta el desarrollo de sistemas de seguridad social amplios que incluyan a toda la población y no solamente a quienes estén asalariados, pasando por el derecho a la salud, a la educación, a la vivienda y al trabajo (Pautassi, 2007).

En esa línea, poner el acento en las cuestiones ligadas al cuidado de las personas se justifica desde la perspectiva de la búsqueda de una mayor igualdad social, en virtud de que la organización de las actividades de cuidado es un aspecto central de los patrones de desigualdad social, principalmente en términos de género. Esto se debe a que, si bien todos y todas debemos ser cuidados/as, las tareas de cuidado están mayoritariamente a cargo de mujeres (Esquivel et al., 2012).

En consecuencia, la forma en que una sociedad encara la provisión de cuidados tiene implicancias significativas para el logro de la igualdad de género, al ampliar las capacidades y opciones de hombres y mujeres o al confinar a las mujeres a los roles tradicionales asociados con la feminidad y la maternidad (Esquivel et al., 2012).

Asimismo, como consecuencia del debate internacional y de la proliferación de un sistema jurídico-administrativo que busca hacer efectiva la igualdad de derechos en todos los escenarios de interacción social, los distintos países disponen de leyes y normativas particulares para esta materia. En especial, han incorporado disposiciones tendientes a la igualdad de género para los casos de la protección de la maternidad y los mecanismos para el cuidado de los niños/as (Pautassi, 2005).

\section{Licencias por maternidad y paternidad: la regulación del cuidado}

Una de las formas de regular el cuidado ha sido a través del establecimiento de sistemas de licencias que permiten -o tienden a permitir- la conciliación de la vida laboral con la vida doméstica. En términos generales, las disposiciones que regulan el ámbito del trabajo remunerado en la Argentina incluyen licencias de diferente extensión con goce de haberes relativos a maternidad y paternidad, y también en casos de adopción, atención de hijos/as menores o con discapacidad y atención del grupo familiar. Adicionalmente, existen beneficios tales como la reducción de jornada laboral para madres de lactantes, y determinadas adecuaciones o cambios en el puesto de trabajo por motivo de gravidez. Sólo unos pocos regímenes laborales incluyen licencias por adaptación escolar o visitas con fines de adopción (Laya y Rossi, s/a).

En el área latinoamericana, las licencias maternales promueven una conciliación de dos esferas -la laboral y la familiar- en un período más 
largo de tiempo. En contraste, las parentales son un instrumento poco conocido y escaso en la región, pero, no obstante, su implementación ha ido avanzando recientemente (Rossel, 2013).

Más allá de las mejoras que podrían exigirse en estos sistemas de licencias, lo que aquí importa es que han significado un inconmensurable avance de índole legislativo en términos de reconocimiento de derechos, que reduce las desigualdades, contribuye ampliamente a la conciliación de la vida laboral con la familiar, y sitúa a las mujeres en una mejor posición en el mercado laboral2.

Actualmente, no existen dudas en cuanto a su importancia, e incluso, están ampliamente extendidas en distintos países del mundo, sólo con variaciones respecto al período de duración del beneficio (Rossel, 2013).

De igual manera, la complejidad del tema está anclada en la multiplicidad de actores, instituciones y sectores que participan en el proceso de cuidado (Esquivel et al., 2012). Los debates sobre cuidados, así son permeados por acciones de incidencia de diversas organizaciones de derechos humanos, la presentación de proyectos legislativos, el debate académico y, en menor medida, a través del accionar del Poder Judicial (Arcidiácono et. al, 2015). A modo de ejemplo, un informe del Equipo Latinoamericano de Justicia y Género (en adelante, ELA) demuestra que, en el período 2008-2013, ha sido sumamente profusa la presentación de proyectos de ley que proponen nuevas regulaciones en torno al tiempo destinado al cuidado, particularmente cambios en el régimen de licencias previsto en la Ley de Contrato de Trabajo (Ley No 20.744, en adelante, LCT). Luego, en el ámbito de la negociación colectiva, que también constituye un importante instrumento para la promoción de la igualdad de oportunidades en el mundo del trabajo, el informe afirma que si bien en el último tiempo las mujeres han logrado avanzar en la participación sindical desde algunos espacios -como las secretarías de género e igualdad de oportunidades o centros de estudio desde donde impulsan debates sobre los derechos de igualdad-la representación femenina sigue siendo escasa en los sindicatos, especialmente en lo que refiere a las posiciones de liderazgo y toma de decisión (Pautassi y Rodríguez Enríquez, 2014).

Frente a esa pluralidad de actores, instituciones y sectores involucrados en la regulación del cuidado, el presente trabajo centra la atención en uno sólo de ellos, las universidades públicas nacionales y, en una dimensión que, en particular, no ha sido analizada con suficiente

\footnotetext{
2 Las críticas en torno a la regulación del cuidado se basan en que la normativa restringe la protección de la madre trabajadora al período de gestación, alumbramiento, posparto y lactancia, sin contemplar la crianza y las demandas de cuidado que tienen los niños en esta etapa. Además, prescinde o bien minimiza la responsabilidad de los varones frente a sus hijos pequeños (Nieves Rico y Pautassi, $2011)$.
} 
atención. Se refiere con ello a las necesidades de cuidado en los casos de estudiantes que se encuentran por fuera de una relación laboral. Las universidades, en ese marco, constituyen un sector que, al delinear políticas públicas ligadas a la educación, cuenta con posibilidades de definir modelos más inclusivos. El estudio se sitúa desde el año 2011 hasta el presente; el recorte temporal está ligado a cuestiones de índole normativas, en virtud de que los primeros antecedentes, hasta donde se ha podido corroborar, datan de aquel año.

A continuación, se describen los avances que conlleva la implementación de sistemas de licencias dentro de la esfera estudiantil, identificando sus beneficios como también sus implicancias en el desarrollo o desempeño académico de quienes se inscriben en las carreras de grado.

\section{Licencias maternales y paternales para universitarios/as: reconociendo derechos}

En el ámbito universitario nacional, resulta importante, en primer lugar, mostrar que se trata de un espacio interesante para su indagación en tanto cuenta con particularidades a destacar. Según un Informe de ELA (Gebruers, 2012) en la Argentina, el espacio universitario posee la particularidad de tener un mayor número de estudiantes mujeres. Asimismo, los datos del Anuario de Estadísticas Universitarias del año 2011 indican que de un total de 1.808.415 estudiantes de universidades e institutos universitarios nacionales y privados de todo el país, el $57,1 \%$ son mujeres. También constituyen el $56,6 \%$ de los nuevos inscriptos, y el $61,2 \%$ de los egresados ${ }^{3}$. En la misma línea, en el último Anuario realizado, esto es, en el año 2013, las cifras se repiten. De un total de 1.830.743 estudiantes, el 57,3\% son mujeres. En cuanto a los nuevos inscriptos, las mujeres representan el $57,2 \%$ y el $61,9 \%$ de los egresados 4 .

Se habla, incluso, de una tendencia general a la feminización de la matrícula. Por un lado, las mujeres mantienen una presencia mayoritaria en las disciplinas históricamente asociadas a su condición (humanidades, servicios sociales), han avanzado en carreras que antes eran prioritariamente masculinas como el derecho y la medicina y siguen estando sub-representadas en carreras con un componente

\footnotetext{
3 Anuario de Estadísticas Universitarias Argentinas 2011, Departamento de Información Universitaria, de la Secretaría de Políticas Universitarias (SPU), Ministerio de Educación de La Nación, Buenos Aires. Disponible en: http://informacionpresupuestaria.siu.edu.ar/DocumentosSPU/Anuario\%20de\%20Estad\% C3\%ADsticas\%20Universitarias\%20-\%20Argentina\%202011.pdf

${ }^{4}$ Anvario de Estadísticas Universitarias Argentinas 2013, Departamento de Información Universitaria, de la Secretaría de Políticas Universitarias (SPU), Ministerio de Educación de La Nación, Buenos Aires. Disponible en: http://informacionpresupuestaria.siu.edu.ar/DocumentosSPU/Anuario_2013.pdf
} 
claramente técnico como son las ingenierías en todas sus variantes o la informática (Pinkasz y Tiramonti, 2006).

Por otra parte, es necesario tener en cuenta otras particularidades propias del espacio universitario argentino. Se trata de un ámbito de formación de mujeres y varones que se hallan en una situación de privilegio relativo, con mayores oportunidades para poder desarrollar todo su potencial en formación, pero que al estar en etapas precisamente de formación, muchos de ellos se encuentran por fuera de una relación laboral formal asalariada y con poca o nula experiencia de trabajo.

Ahora bien, a pesar de que el ámbito universitario es, por las razones expuestas, un espacio relevante para la indagación desde la perspectiva feminista, la licencia estudiantil por maternidad y paternidad para estudiantes no es una cuestión muy frecuente en la investigación académica. Una explicación posible consiste en que los estudios e investigaciones desarrollados habitualmente toman como referencia la relación de la mujer con el mercado laboral. Sin embargo, como los estudiantes se encuentran en un período de formación que, en muchos casos, los sitúa por fuera de este mercado, podría decirse que siempre ha existido un vacío en relación a los mismos 5 . Hasta el año 2011 aproximadamente, los universitarios sólo gozaban formalmente de licencias cuando eran empleados/as por la LCT.

Adicionalmente, en algunas ocasiones existen jóvenes madres y padres que participan en la vida universitaria sin contar con un amplio apoyo de las instituciones donde llevan a cabo sus estudios. La disponibilidad de tiempo, dinero y recursos de las familias para el cuidado de niños y niñas pequeños incide directamente en la formación y en el desempeño laboral o profesional de los estudiantes/as.

Sin embargo, desde el año 2011, el tema ha empezado a ocupar un lugar más visible en la agenda universitaria en virtud de la creación reciente de un marco normativo para las licencias estudiantiles en algunas universidades nacionales. En ese contexto, casas de estudios de diferentes regiones del país han incorporado sistemas de licencias para los y las estudiantes. Así, se encuentra el Reglamento de justificación de inasistencias y de licencias especiales para estudiantes sancionado por la Universidad Nacional de La Pampa según Resolución 248/2011 y su posterior modificación (2016); el sistema de licencias de la Facultad de Psicología de la Universidad Nacional de Tucumán según Resolución № 206-8-2007; el régimen de licencias de la Universidad Nacional de Córdoba según Ordenanza $\mathrm{N}^{\circ} 2$ del año 2011; el reglamento de licencias estudiantiles de la Universidad Nacional de Salta según Resolución del Consejo Superior 502/2012; el sistema de licencias

5 Se han efectuado numerosas investigaciones sobre las mujeres que parten de su relación con el mercado laboral. En esa línea, ver: (ELA, s/a) y (Pautassi y Rodríguez Enríquez, 2014). 
estudiantiles de la Universidad Nacional de Cuyo según Ordenanza $N^{\circ}$ 39 del año 2013; el régimen de licencias estudiantiles de la Universidad Autónoma de Entre Ríos según Ordenanza № 62 del 2015; el régimen de licencias y justificaciones estudiantiles de la Universidad Nacional de La Rioja según Ordenanza № 47 del año 2015, entre otros6.

Dentro de las licencias previstas, algunas destacan por estar directamente ligadas a las necesidades de cuidado y vienen a cubrir el vacío que se mencionó para los estudiantes que están por fuera del mercado laboral. En primer lugar, una licencia implementada por todos los sistemas enumerados y que resulta de especial interés en el presente: la licencia por maternidad. La misma ofrece la posibilidad a las madres de ausentarse de sus obligaciones académicas por un período de tiempo que varía según el reglamento, antes y después del nacimiento del niño/a7. En segundo lugar, otras entidades educativas avanzaron aún más allá e incluyen la licencia por paternidad (La Pampa, Córdoba, Cuyo, La Rioja), que habilita a los padres a ausentarse por un período considerablemente menor que el de la madre ante el nacimiento del hijo/a ${ }^{8}$. También, se han incorporado licencias por adopción (La Pampa, Cuyo, Tucumán, Entre Ríos, La Rioja) que se aplican tanto a las madres como padres que hayan obtenido la guarda con fines de adopción, y licencias para la atención del grupo familiar, que refieren a cualquier persona que tenga un miembro de su grupo familiar enfermo, accidentado o que requiera una determinada atención (La Pampa, Córdoba, Cuyo, Tucumán, Entre Ríos, La Rioja)?9

6 También es dable mencionar que, en la esfera legislativa, la senadora nacional Sonia Escudero por la provincia de Salta (2001-2013) presentó en el año 2013 un proyecto de ley cuyo fin era obligar a las instituciones educativas de los niveles primarios, secundarios, terciarios y universitarios a que establezcan licencias por maternidad para sus estudiantes. Ello, tal como se afirmaba en su artículo 1, para garantizar el acceso a la educación y a la igualdad de oportunidades entre sus estudiantes de sexo femenino. Disponible en: http://www.senado.gov.ar/parlamentario/parlamentaria/avanzada

7 Para el presente trabajo, el término licencia por maternidad incluye las licencias por embarazo y posparto.

8 Mientras que a las madres se les otorga una licencia que varía entre 45 días antes del parto y hasta 150 días corridos después del nacimiento, según cada reglamento, generalmente la licencia por paternidad sólo contempla 15 días posteriores al nacimiento del niño/a. A modo de ejemplo, ver el sistema de la Universidad Nacional de Cuyo según Ordenanza $N^{\circ} 39$ del año 2013 y el régimen de la Universidad Nacional de La Rioja según Ordenanza № 47 del año 2015.

9 Además, las universidades difieren en los sistemas con algunas licencias especiales también vinculadas al cuidado, con distinciones en el nombre o categoría. A modo de ejemplo, la Universidad Nacional de Córdoba cuenta con una licencia particular para el caso de hijo/a con discapacidad (artículo 1, inc. d ordenanza N 2/2011). La Universidad Nacional de Cuyo posee una licencia específica para la atención a hijos menores (artículo 3, inc. h.2 Ordenanza No 39/2013) al igual que la Facultad de Psicología de la Universidad Nacional de Tucumán (ap. II, art. 1, inc. e Resolución No 206-8-2007), la Universidad Nacional de Entre Ríos (art. 4, inc. e, Ordenanza № 62/2015) y la Universidad Nacional de La Rioja (artículo 2, inc. h.2, Ordenanza No 47/2015) 
El objeto principal de todas estas licencias radica, entonces, en la suspensión de los plazos de actuación de quienes soliciten el beneficio, manteniéndose en consecuencia, en estos casos, las condiciones académicas e institucionales obtenidas en el momento del otorgamiento de la licencia y por el período previsto.

Estos sistemas, a pesar de las variaciones en cuanto a sus alcances y dimensiones, promueven una perspectiva de género delineando una concesión de licencias que permite avances en términos de igualdad y equidad. Se trata, fundamentalmente, del reconocimiento de un derecho más para los universitarios, ya otorgado previamente al personal docente y no docente de las casas de estudios. Importa, asimismo, la afirmación del cuidado como derecho, al permitir que el/la estudiante pueda ausentarse de sus actividades por un período de tiempo determinado en el cual debe cumplir con ciertas exigencias ligadas a cuestiones de cuidado sin perjudicar su desempeño académico.

Pautassi (2007) explica que, si bien no toda desigualdad implica discriminación, en virtud de que la garantía de igualdad no requiere el trato igualitario a quienes se encuentran en diferentes circunstancias, de allí resulta que las desigualdades en el mundo de las relaciones de cuidado suelen verse, en muchos casos, como una discriminación encubierta o bien simplemente no verse y asumirse como parte del nuevo escenario (Pautassi, 2007). De una u otra manera, tradicionalmente ha existido una discriminación -sea encubierta o no percibida - en las universidades nacionales que ha perjudicado a determinados/as estudiantes que debían asumir obligaciones de cuidados en cuanto a su desempeño y/o continuidad académica. Es por ello que, en el marco de este derecho al cuidado de los y las estudiantes, estos sistemas vienen a romper con esa discriminación, avanzando hacia un modelo educativo más inclusivo.

Asimismo, las características distintivas del sistema universitario mencionado nos conducen a puntualizar la relevancia social de las medidas adoptadas por las universidades nacionales, verfebradas como respuestas para que su población estudiantil pueda satisfacer sus derechos y necesidades vinculadas al cuidado, en razón de estar enfrentando etapas de crianza y cuidado de sus hijos/as mientras cursan sus carreras en ta universidad. Así, los sistemas de licencias implementados por los centros educativos aducidos conforman un destacable esfuerzo por superar las desigualdades existentes en la actualidad entre el alumnado universitario. Configuran una respuesta al reclamo por la igualdad formal, que elimina las barreras legales existentes hacia el desarrollo pleno de las capacidades de las mujeres (Pautassi, 2005).

Lamentablemente, no es posible saber con exactitud cuántos estudiantes se encuentran en situación de maternidad/paternidad 
durante sus carreras universitarias ya que no existen estadísticas al respecto. Incluso, muchos/as estudiantes abandonan los estudios ante las dificultades de continuar con la carrera durante el embarazo/maternidad y esa situación no queda registrada o bien se registra como deserción universitaria. No obstante, tanto los centros estudiantiles como también los centros de cuidado para la primera infancia (jardines, guarderías), reconocen la considerable demanda por parte de los y las estudiantes ${ }^{10}$. Es posible que, de avanzar todas las instituciones hacia la implementación del sistema de licencias, pueda efectuarse una sistematización de datos que permita dar cuenta de la problemática en términos reales.

\section{El fundamento jurídico-normativo: bases para la implementación del sistema}

La necesidad de implementar sistemas de licencias para los y las estudiantes universitarios encuentra su raíz en el reconocimiento del derecho a la educación como derecho fundamental incluido en los instrumentos de derecho internacional incorporados a la Carta Magna de la Argentina. Así, el artículo 13 inc. 2 del Pacto Internacional de Derechos Económicos, Sociales y Culturales establece que "los Estados Partes [...] reconocen el derecho de toda persona a la educación"; por su parte, el artículo 26 de la Declaración Universal de Derechos Humanos dispone que "la educación tendrá por objeto el pleno desarrollo de la personalidad humana y el fortalecimiento del respeto a los derechos humanos y a las libertades fundamentales".

Incluso, ya la Ley Nacional de Educación Superior, según Decreto No 24521/95, enfatiza en su articulado la necesidad de garantizar el derecho a la educación en base a la igualdad de oportunidades. De esa forma, establece en su artículo 4 inc. e, que son objetivos de la educación superior "profundizar los procesos de democratización en la educación superior, contribuir a la distribución equitativa del conocimiento, y asegurar la igualdad de oportunidades". La misma norma, en su artículo 13, inc. c, instituye que los estudiantes de las instituciones estatales de educación superior tienen derecho a "obtener

10 En el marco del Proyecto "Una aproximación socio jurídica a las respuestas de las universidades nacionales a las necesidades de cuidado en la primera infancia. Un estudio de caso centrado en el sistema de licencias y de servicios de cuidado para los/as estudiantes universitarios", dirigido por Carla Zibecchi, se efectuaron entrevistas que aportaron la información mencionada. Entre ellos, se entrevistó al Presidente del centro de estudiantes de la Facultad de Derecho de la Universidad de Buenos Aires (UBA) bajo la dirección de Franja Morada; a la Directora del Jardín Maternal de la Facultad de Derecho de la UBA; al Secretario de Extensión, Cultura Científica y Bienestar de la Facultad de Ciencias Exactas y Naturales, a cargo de la supervisión y coordinación del Jardín Maternal de la Facultad; a un ex consejero estudiantil por la agrupación Franja Morada de la Universidad Nacional de La Pampa (UNLPam); y, por último, a la Directora de Acción Social de la Secretaría de Bienestar Universitario de la UNLPam. 
becas, créditos y otras formas de apoyo económico y social que garanticen la igualdad de oportunidades y posibilidades, particularmente para el acceso y permanencia en los estudios de grado [...]".

La normativa descripta no sólo permite, sino que induce a las entidades e instituciones educativas a la incorporación de medidas que tiendan a la promoción de la educación universitaria en condiciones de igualdad, encontrándose allí incluida la implementación del sistema de licencias referido. Se puede afirmar, entonces, que las licencias objeto del presente artículo se verían fundamentadas en tres derechos universales y esenciales: educación, igualdad y cuidado, recordando que, como se mencionó, desde una perspectiva de género se pretende avanzar hacia el reconocimiento del cuidado como derecho universal ${ }^{11}$.

A modo de ejemplo, en el plano internacional una serie de estatutos universitarios muestran, en el mismo sentido, el progresivo reconocimiento de los derechos de los y las estudiantes. Así, principalmente en Inglaterra, universidades como Oxford, Cambridge, Leeds, Notthingham, Birmingham, King's College, Glasgow, London School of Economic and Political Science, entre otras, cuentan con previsiones normativas que regulan de manera detallada y precisa los casos de estudiantes madres o padres que cursan en aquellas unidades educativas, otorgándoles tanto licencias como otros derechos vinculados al cuidado ${ }^{2}$.

Después de mostrar los fundamentos jurídico-normativos para la existencia de los sistemas en cuestión, junto a los beneficios en términos de igualdad y reconocimiento de derechos, se ahondará, a continuación, en el sistema específico de licencias implementado por la Universidad Nacional de La Pampa.

11 Si bien el derecho al cuidado no está reconocido explícitamente por los tratados y Pactos Internacionales de Derechos Humanos, sí está incorporado en el marco de derechos que otorga el Sistema Internacional de Derechos Humanos. Frente a ello, sólo habría que promover instancias de monitoreo y de exigibilidad a cada uno de los Estados (Pautassi, 2007). En esa línea, ver Pacto Internacional de Derechos Económicos, Sociales y Culturales (PIDESC), la Convención sobre la Eliminación de Todas las formas de Discriminación contra la Mujer (CEDAW), la Convención sobre los Derechos del Niño (CDN), entre otros.

12 La creación de los Estatutos que otorgan estos derechos estuvo marcada por la sanción de la Ley de Igualdad 2010 (Equality Act, 2010) que fortaleció significativamente la protección de los y las estudiantes durante el embarazo y la maternidad en Inglaterra, Escocia y Gales. Además, a modo de ejemplificar las desigualdades sufridas por el alumnado que enfrenta la maternidad/paternidad, una investigación realizada por la Unión Nacional de Estudiantes (NUS según sus siglas en inglés) sobre la experiencia de los/las estudiantes que tenían niños en la educación superior en el Reino Unido, mostró que el 59\% de los encuestados que habían ejercido su rol de madres/padres durante sus estudios no se habían sentido apoyadas/os por sus universidades (NUS, 2009). Las estudiantes embarazadas han tenido que enfrentar barreras como ser obligadas a retirarse de su curso e incluso verse impedidas de rendir los exámenes (Pung, 2010). 


\section{Una normativa específica: el sistema de licencias de la Universidad Nacional de La Pampa}

La Universidad Nacional de La Pampa, mediante la Resolución 248 del Consejo Superior del año 2011, instituyó un reglamento general de licencias estudiantiles inclusivo de todos los y las estudiantes de la universidad que merece una especial atención. En el cuerpo de la misma, pueden identificarse tres pilares que fundamentan la implementación del sistema de licencias: i) la protección de la educación y la salud como derechos consagrados constitucionalmente; ii) su definición como una herramienta económicamente viable, y iii) su presentación como respuesta al reclamo estudiantil.

En primer lugar, la normativa afirma que "el régimen de licencias propuesto configura un género de ayuda destinado a estudiantes que no pueden desarrollar normalmente sus actividades curriculares por atravesar una situación personal de fuerza mayor". En ese sentido, el instrumento reconoce que tanto la Educación Pública como la Salud son derechos consagrados constitucionalmente; conforman "pilares fundamentales para el progreso en una sociedad democrática, así como también, elementos trascendentes para la movilidad social de todos los ciudadanos y objeto de política gubernamental". Ante ello, como consecuencia, se reconoce el deber de la institución de arbitrar los mecanismos aptos para lograr una mayor democratización de las oportunidades y posibilidades para los y las estudiantes, de manera que accedan, avancen y concluyan exitosamente sus estudios.

En la misma línea, afirma que uno de los deberes de toda universidad es el de garantizar los principios y garantías insertos en nuestra Carta Magna y en los Tratados Internacionales, muchos de los cuales poseen la misma jerarquía que aquella (según el artículo 75, inc. 22 de la Constitución Nacional), entre ellos, la igualdad de oportunidades y posibilidades, la no discriminación, el derecho de enseñar y aprender y el acceso a la educación superior. Incluso, defiende que "la Corte Suprema de Justicia de la Nación ha entendido en numerosos pronunciamientos que los derechos y garantías constitucionales deben ser entendidos en un sentido amplio".

En segundo lugar, un aspecto de particular importancia, consiste en la afirmación de que ese sistema de licencias estudiantiles "es una herramienta económicamente viable, que no genera erogaciones extraordinarias". De alguna manera, ello demuestra o enfatiza la inviabilidad de utilizar argumentos vinculados a los costos monetarios de la implementación de la figura para desvalorizarla. Es sabido que una de las principales barreras que deben enfrentarse para el impulso de nuevos proyectos en el ámbito público está definida por el costo económico de la medida a implementar, y por ello, el segundo pilar se establece como un aspecto elemental. 
En tercer lugar, se encuentra el último pilar, definido por el reconocimiento de que la medida creada junto a sus previsiones proviene como consecuencia de un reclamo por parte de la comunidad estudiantil. En una entrevista realizada al ex Consejero Estudiantil de la Agrupación Franja Morada ante el Consejo Superior de la universidad durante el año 2010, el estudiante de Abogacía que presentó el proyecto del Reglamento, expresó que el reclamo provino de casos puntuales e individualizados que llevaron a la visualización de la problemática. Fueron los y las estudiantes que enfrentaban dificultades personales en el transcurso académico quienes acercaron y compartieron sus inquietudes y generaron que, en efecto, surja la propuesta de creación de un sistema de licencias que cubriera a los y las alumnos/as ante determinadas situaciones ${ }^{13}$.

Si bien, como se dijo, surge en el año 2011, recientemente el Consejo Superior aprobó la Resolución No 015 del 17 de febrero de 2016, por la que se deroga la Resolución anterior y se establece un nuevo Reglamento General de Licencias Estudiantiles acorde a las modificaciones impuestas por el nuevo Código Civil y Comercial de la Nación"14. En la misma, el artículo 2 dispone que la universidad "otorgará Licencias Estudiantiles a los estudiantes que por diversas causas atraviesen contingencias extraordinarias que impidan el normal desarrollo de las actividades propias de un estudiante universitario". Dentro de esas situaciones, se encuentra el tipo de licencia por maternidad o paternidad (art. 9, inc. A), por adopción o guarda preadoptiva con fines de adopción (art. 9 inc. b), y la licencia por atención del grupo familiar (art. 9 inc. g), entre otras ${ }^{15}$.

En todos los casos, el efecto principal de la prestación de las licencias será la suspensión de los plazos de la actuación universitaria para los que hayan solicitado el beneficio, manteniéndoseles las condiciones académicas e institucionales obtenidas en el momento del otorgamiento ${ }^{16}$. Además, una particular característica del sistema normado consiste en la concesión de licencias retroactivas en los casos en que extraordinariamente el hecho fundante de la causal fuere imprevisible y súbito, pudiendo allí solicitar que el otorgamiento de la licencia sea con efecto retroactivo al día del acaecimiento de la causal 17 .

13 Ex Consejero Estudiantil de la universidad por la agrupación Franja Morada, comunicación personal, 24 de julio de 2016.

14 A partir del 1 de agosto de 2015 entró en vigencia el nuevo Código Civil y Comercial de la Nación, aprobado en la Ley 26.994 del año 2014.

15 También se incluyen los supuestos de licencias por afecciones o lesiones de corto tratamiento o baja complejidad; afecciones, enfermedades e intervenciones quirúrgicas de alta complejidad; donación de órganos; y fallecimiento de familiar, cónyuge y/o quien cohabite en unión convivencial (art. 9).

16 Artículo 2, Resolución 15/2016.

17 Artículo 30, Resolución N 248/2011. 


\section{Consideraciones finales}

Ya desde el comienzo, se enfatizó la importancia de considerar el cuidado como un derecho universal. Partiendo de ello, la conceptualización del cuidado como un derecho conlleva para el Estado no sólo obligaciones negativas, como abstenerse de entorpecer los servicios de guarderías infantiles, sino también obligaciones positivas, tales como proveer los medios necesarios para poder cuidar y garantizar que el cuidado se lleve adelante en condiciones de igualdad (Gherardi y Zibecchi, 2011).

En ese marco, la implementación de licencias por maternidad y paternidad ha reflejado un inconmensurable avance por parte del Estado dentro de esas obligaciones positivas; ha logrado una mayor conciliación de la esfera laboral y la doméstica-familiar, y ha creado una base normativa para la protección de madres y padres que llevan a cabo tareas y obligaciones de cuidado.

En la misma línea, este trabajo ha hecho especial hincapié en el contexto universitario nacional, intentado mostrar cómo algunas de las universidades argentinas han permitido un progreso en términos de equidad que favorece la superación de problemáticas que no se encontraban lo suficientemente visibilizadas. En especial, se ha destacado el régimen establecido por la Universidad Nacional de La Pampa por ser una de las primeras en implementar un sistema de licencias entre las instituciones universitarias del país.

Al indagar en ese ámbito, pudo verse que el número de mujeres estudiantes universitarias no representa la minoría del alumnado y, por el contrario, los datos parecen revelar un posible incremento de las cifras femeninas dentro del ámbito académico en las estadísticas de un futuro cercano. Ello, sumado a los beneficios que acarrea el novedoso régimen de licencias, reforzó el argumento a favor de su implementación.

No existen dudas acerca de la importancia de garantizar el derecho a la educación en condiciones de igualdad en el mundo contemporáneo. Ante ello, los sistemas descriptos conforman una viable herramienta para el reconocimiento progresivo de derechos de los y las estudiantes sin ningún tipo de discriminación. Tomando en consideración los derechos a la educación, la igualdad y el cuidado, los regímenes de licencias permiten garantizar los tres elementos en simultáneo, creando un sistema que merece especial reconocimiento.

Seguir permitiendo las barreras en el acceso o la permanencia de los y las estudiantes universitarios implica continuar reafirmando y definiendo estereotipos sociales respecto de las expectativas acordes al desempeño de mujeres y los hombres en las sociedades actuales en la esfera pública (Pautassi, 2005). 
Debe avanzarse hacia la incorporación masiva del tema en la agenda universitaria, generando una sanción normativa que construya mecanismos institucionales de género. Además, es importante que la adopción de los sistemas de licencias por maternidad y paternidad para estudiantes se lleve a cabo por el simple hecho de serlo, reconociendo las implicancias que pueden acarrear por vivir situaciones de crianza y cuidado.

De uno u otro modo, parecería ser una deuda de las universidades nacionales, y con ello, del Estado, la falta de reconocimiento de este derecho a todos los y las estudiantes que, a pesar de tener que enfrentar obligaciones y responsabilidades propias de la maternidad y paternidad, eligen continuar sus estudios apostando a la educación como derecho irrenunciable. El apoyo por parte de las Casas de Estudios resulta fundamental para poder avanzar hacia una sociedad más equitativa, educada y democrática.

Por último, si bien la creación del marco normativo que contempla las licencias en la esfera universitaria marca un destacado avance en materia de género, parece ser un proceso inicial que demandará varios años hasta que alcance un nivel de implementación más afianzado. Para ello, debe lograrse que los mismos estudiantes identifiquen y reconozcan sus propios derechos, exigiendo en consecuencia el cumplimiento de los mismos, proceso que demandará tiempo y puede enfrentar obstáculos o barreras de parte de las autoridades educativas. Debería avanzarse, sin ninguna duda, hacia su total implementación, para dejar de condicionar la inserción de mujeres y hombres en el ámbito profesional y poder lograr una conciliación cada vez mayor de la vida laboral con la familiar.

\section{Referencias bibliográficas}

Arcidiácono, P., Bestard, A., Riesco, R. y Zibecchi, C. (2015). Asignaciones familiares, licencias e infraestructura de cuidado. Avances en la intervención judicial. Revista Perspectivas de Políticas Públicas, 5(9), 117 137.

Borderías, C., Carrasco, C. y Torns, T. (eds.) (2011). El trabajo de cuidados. Historia. Teoría y Políticas. Madrid: Catarata.

Carrasquer Oto, P. (2013). El redescubrimiento del trabajo de cuidados: algunas reflexiones desde la sociología. Cuadernos de Relaciones Laborales, 31(1), 91-113. 
ELA (s/a). Licencias. Tiempos para cuidar. Equipo Latinoamericano de Justicia y Género, Oficina Argentina del Fondo de Naciones Unidas para la Infancia (UNICEF) y el Centro de Implementación de Políticas para la Equidad y el Crecimiento (CIPPEC).

Equality Act (2010). Equality Act 2010. HM Stationery Office. London. Descargado de http://www.legislation.gov.uk/ukpga/2010/15/pdfs/ukpga_20100015_en. pdf

Esquivel, V., Faur, E. y Jelin, E. (eds.) (2012). Las lógicas del cuidado infantil. Entre las familias, el Estado y el mercado. Büenos Aires: IDES.

Esquivel, V. (2015). El cuidado: de concepto analítico a agenda política, Revista Nueva Sociedad, 256, 63-74.

Gebruers, C. (2012). Acoso sexual en espacios educativos en Argentina. Una aproximación a su regulación y abordaje a partir de la revisión de decisiones judiciales. Serie Documentos de Trabajo. Equipo Latinoamericano de Justicia y Género (ELA). Buenos Aires. Descargado de www.ela.org.ar.

Gherardi, N. y Zibecchi, C. (2011). El derecho al cuidado: ¿ Una nueva cuestión social ante los tribunales de justicia de Argentina? Revista Política, 49(1), 107-138.

Laya, A. y Rossi, F. (s/a). Aportes para la discusión legislativa sobre reformas necesarias en materia de cuidado. Documentos de Trabajo "Políticas Públicas y Derecho al Cuidado" 1, ELA- Equipo Latinoamericano de Justicia y Género, Buenos Aires. Descargado de http://elcuidadoenagenda.org.ar/wp-content/uploads/2014/12/DT-1Aportes-para-la-discusion-legislativa.pdf

Nieves Rico, M. y Pautassi, L. (2011). Licencias para el cuidado infantil. Derecho de hijos, padres y madres. En CEPAL, Cuidado infantil y licencias parentales. Boletín de la infancia y adolescencia sobre el avance de los Objetivos de Desarrollo del Milenio, 12, 4-9.

National Union of Students (2009). Meet the parents: the experience of students with children in further and higher education. London. Descargado de https://www.nus.org.uk/global/nus_sp_report_web.pdf/

Pautassi, L. (2005). Legislación laboral y género en América Latina. Avances y omisiones. Reunión de expertos "Políticas hacia las familias, protección e inclusión sociales". CEPAL, 28 y 29 de junio de 2005. Descargado de http://dds.cepal.org/eventos/presentaciones/2005/0628/Laura_Pautassi. pdf

Pautassi, L. (2007). El cuidado como cuestión social desde un enfoque de derechos. Serie Mujer y Desarrollo No 87, Santiago de Chile: CEPAL. Descargado de 
http://repositorio.cepal.org/bitstream/handle/1 1362/5809/S0700816_es.p df; ;sessionid=3D79C5CABB6CA3CF263FD69A8D2DF33A? sequence $=1$

Pautassi, L. (2013). Perspectivas actuales en torno al enfoque de derechos y cuidado. La autonomía en tensión. En Pautassi, L. y Zibecchi, C. (Comp.), Las fronteras del cuidado. Agenda, derechos e infraestructura (pp. 99-132). Buenos Aires: Editorial Biblos.

Pautassi, L. y Rodríguez Enríquez, C. (2014). La organización social del cuidado en niños y niñas. Elementos para la construcción de una agenda de cuidados en Argentina. Buenos Aires: ADC-CIEPP-ELA.

Paura, V. y Zibecchi, C. (2014). Dinámicas institucionales, lógicas de los actores y territorio en el estudio de la política social. Veinte años de investigación en la Argentina. En Pautassi, L. (Dir), Marginaciones sociales en el área metropolitana de Buenos Aires. Acceso a la justicia, capacidades estatales y movilización legal (pp. 333-396). Buenos Aires: Editorial Biblos.

Pinkasz, D. y Tiramonti, G. (2006). Las oportunidades educativas de las mujeres en la modernización de los 90 en Argentina. En FLACSO, IESCO y Hexagrama Consultoras, Equidad de Género y Reformas educativas: Argentina. Chile. Colombia. Perú (pp. 51-95). Santiago de Chile: Hexagrama Consultoras. Descargado de http://www.oei.es/reformaseducativas/equidad_genero_reformas_educ ativas.pdf

Pung, E. (comp.) (2010). Student pregnancy and maternity: implications for higher education institutions. Equality Challenge Unit. November 2010. Descargado de http://www.ecu.ac.uk/publications/student-pregnancyand-maternity/

Rossel, C. (2013). Políticas para las familias en América Latina: Panorama de políticas de reducción de pobreza y conciliación entre trabajofamilia. Naciones Unidas. Descargado de http://www.un.org/esa/socdev/family/docs/FAMILYPOLICIESINLATINAME RICA.pdf 